\title{
Arbor
}

\section{Introducción a la lógica cuántica}

\section{Adán Cabello*}

Arhor CLXVII, 659-660 (Noviembre-Diciembre 2000), 489-507 pp.

En este trabajo trataremos de responder las siguientes preguntas: 1. ¿Qué entendemos por «lógica» de una teoría física? 2. ¿Cuál es la lógica de la fisica clásica? 3. ¿Cómo se puede construir una lógica basada en la mecánica cuántica? 4. ¿Para qué sirve y para qué podría servir la lógica cuántica? Para ello, empezaremos estudiando la lógica de un sistema clásico sencillo. Después introduciremos la lógica cuántica propuesta por Birkhoff y von Neumann. Terminaremos describiendo tres ejemplos (el teorema de Kochen-Specker, el programa de las escuelas de Harvard y Ginebra, y el programa de Putnam) que ilustran los éxitos, expectativas y fracasos de la lógica cuántica.

\section{Introducción}

John Bell, uno de los más penetrantes investigadores de la mecánica cuántica (MC), decía que todo el vasto campo de la lógica cuántica (LC) había surgido por el mal uso de la palabra «medición» [1]. Hablar del "resultado de una medición" sugiere que éste revela alguna propiedad preexistente. Si uno piensa así, entonces es razonable esperar que los resultados de los experimentos cuánticos obedezcan la lógica común, y resulta sorprendente descubrir que no lo hacen. Esta visión del origen de la LC es demasiado simplista. Bell creía que los resultados de los experimentos cuánticos estaban determinados por el estado del sistema físico

(*) e-mail: adan@cica.es 
sobre el que se realiza la medición y por el estado del aparato de medida, y que cuando se tuviese en cuenta este último, las dificultades de interpretación de la MC cesarían y la llamada LC se convertiría en la lógica común que rige en los sistemas físicos clásicos. Hoy la opinión mayoritaria entre los físicos es que esto no es así. Hoy creemos que los resultados de los experimentos cuánticos no están predeterminados de ninguna manera, ni siquiera por el estado conjunto sistema-aparato de medida, y que la MC es una teoría completa en el sentido de que dice todo lo que se puede decir sobre los resultados de una "medición". En cuanto a la LC, su historia es tan rica, que hacer un simple recuento de todas las tentativas para desarrollar «lógicas no-clásicas» inspiradas en la MC sería una labor titánica. Hemos pues de limitar nuestras aspiraciones.

En este trabajo nos conformaremos con dar respuesta a las siguientes preguntas: (1) ¿Qué queremos decir cuando hablamos de la «lógica» de una teoría física? (2) ¿Cuál es la lógica de la física clásica? (3) ¿Cómo se puede construir una lógica basada en la MC? (4) ¿Para qué sirve y para qué podría servir en el futuro la LC?

La lógica, en general, estudia la estructura de los enunciados de un lenguaje, con independencia de su contenido. Investiga una serie de nociones - consistencia, validez, implicación y similares- aplicadas a tales enunciados. Como herramientas, usa extensamente la simbolización y el álgebra; en particular, investiga qué estructuras algebraicas poseen los conjuntos de enunciados.

En la lógica de una teoría física, el «lenguaje» está formado por todos los posibles enunciados permitidos por esa teoría acerca de las propiedades o de los resultados de los experimentos sobre un sistema físico. Todo esto quedará más claro a medida que avancemos.

En este trabajo llamaremos lógica clásica a la estructura algebraica característica del conjunto de las proposiciones clásicas asociadas a un sistema físico clásico (i.e., cuya descripción no requiere la $\mathrm{MC}$ ). Tal estructura captura de una manera esencial los conceptos de "magnitud física», "propiedad", y «espacio de fases" propios de la física clásica. En la Sec. II veremos cuál es la lógica de un sistema clásico sencillo. Con ello perseguimos: (a) Entender cuáles son los objetivos de la lógica, cómo trabaja y qué elementos maneja. (b) Introducir notación y vocabulario. (c) Ver en qué sentido una estructura algebraica permite capturar de manera esencial la información que proporciona la física clásica sobre un sistema particular. (d) Tener una referencia que nos permita establecer cuáles son las diferencias entre la lógica clásica y la LC.

Bajo el nombre de lógica cuántica o aproximación lógico-algebraica a la $\mathrm{MC}$ se reúnen diversos esfuerzos teóricos que tienen en común el ob- 
jetivo de lograr una síntesis algebraica de la $\mathrm{MC}$, o bien que hacen uso de estructuras algebraicas para abordar ciertos problemas de la MC, en particular su interpretación, su relación con la física clásica, y la posibilidad de que exista una teoría más general. La idea que subyace en esas tentativas es que cualquier teoría física se caracteriza no sólo por su formulación matemática, sus relaciones epistemológicas, y su interpretación, sino también por un aspecto que ha pasado casi siempre desapercibido: su estructura algebraica. Así, estos esfuerzos buscan las soluciones a los problemas de la MC investigando sus estructuras lógicas.

La LC nace con un trabajo de Birkhoff y von Neumann publicado en 1936. En la Sec. III describiremos este trabajo y las estructuras algebraicas a que da lugar. Esa sección es bastante técnica y requiere cierta familiaridad con el formalismo habitual de la MC.

El trabajo de Birkhoff y von Neumann estimuló, especialmente en los años 60 y 70, todo un programa de investigación. En un intento de resumir los objetivos y el desarrollo de este programa - de ramificaciones laberínticas-, he elegido tres ejemplos que, en mi opinión, ilustran los éxitos, expectativas y fracasos de la LC, y he tratado de sintetizar el estado actual de cada una de esas empresas.

El teorema de Kochen y Specker, del que nos ocuparemos en la Sec. IV A es un ejemplo de cómo la LC impone severas limitaciones a las posibles interpretaciones de la MC. En este sentido, es un «éxito» de la LC (aunque se puede reformular y entender sin necesidad de ella).

El trabajo de las escuelas de LC de Harvard (representada por Mackey y Maczyński) y Ginebra (representada por Finkelstein, Jauch y Piron), del que trata la Sec. IV B, es un ejemplo de un programa incompleto inspirado en la LC de Birkhoff y von Neumann. Estas escuelas pretenden obtener una reformulación algebraica "completa" de la MC, con la esperanza de que tal reformulación - de existir-proporcione pistas sobre las estructuras lógicas de una - todavía inexistente- teoria más general. Es un ejemplo de qué cosas quedan por hacer en LC.

Por último, el trabajo de Putnam (de 1969), del que nos ocuparemos en la Sec. IV C, es un ejemplo de cómo la LC ha suscitado determinadas expectativas que finalmente han quedado sin concreción clara o se han demostrado infundadas.

Más allá de sus éxitos y fracasos, hoy por hoy la LC constituye una formuláción (incompleta) de la MC, alternativa a la usual mediante espacios de Hilbert, y como tal es un marco natural en el que estudiar ciertos problemas.

Finalmente, en la Sec. V, sugeriremos una serie lecturas adicionales para profundizar en los diferentes aspectos de la LC. 


\section{Adán Cabello}

\section{La lógica de la física clásica}

\section{A. La lógica de las proposiciones de un sistema clásico sencillo}

Para entender cuáles son los objetivos y las herramientas de la lógica aplicada a las teorías físicas, vamos a empezar por estudiar la lógica de un sistema clásico. Consideremos un sistema sencillo formado por dos interruptores (a los que llamaremos "primero" y "segundo"), cada uno de los cuales sólo puede estar o «encendido» o «apagado». Una proposición clási$c a$ es un enunciado - necesariamente "verdadero» o «falso»- acerca de las propiedades del sistema. Por ejemplo, una proposición es «el primer interruptor está encendido" (proposición que en esta sección denotaremos por $A$ ), otra es «el segundo interruptor está encendido» (proposición que denotaremos por $B$ ). Además, esas proposiciones están ligadas por los signos, $\neg, \wedge \mathrm{y} \vee$ (que reciben el nombre de functores lógico-proposicionales $o$ functores veritativos), que representan, respectivamente, "negación" (por ejemplo, $\neg A$ representa la proposición «el primer interruptor no está encendido»), "conjunción» (por ejemplo, $A \wedge B$ representa la proposición «el primer interruptor está encendido $y$ el segundo interruptor está encendido»), $\mathrm{y}$ «disyunción no excluyente» (por ejemplo, $A \vee B$ representa la proposición «el primer interruptor está encendido $o$ el segundo interruptor está encendido", que sólo es falsa si ambos interruptores están apagados).

Por otro lado, el estado del sistema se puede caracterizar mediante un punto en un espacio de fases $S$. Un hecho interesante es que cada una de las proposiciones se puede identificar como un subconjunto de $S$. En nuestro ejemplo, el espacio de fases puede ser un simple cuadrado dividido mediante dos ejes cartesianos en cuatro cuadrantes. La proposición $A$ se puede identificar con los dos cuadrantes que están por encima del eje $x$, subconjunto de $S$ que llamaremos $S_{A}$. De igual modo, $\neg A$ se puede identificar con los dos cuadrantes que están por debajo del eje $x$, subconjunto que llamaremos $\bar{S}_{A}$. $B$ se puede identificar con los cuadrantes que están a la derecha del eje $y$, subconjunto que llamaremos $S_{B} . \neg$ se puede identificar con los cuadrantes que están a la izquierda del eje $y$, subconjunto que llamaremos $\bar{S}_{B}$. De hecho, siempre es posible identificar cualquier subconjunto imaginable de $S$ con una proposición. Recíprocamente, cualquier proposición imaginable siempre se puede representar mediante un subconjunto de $S$. Por ejemplo, el subconjunto $S_{A} \cap S_{B}$ representa la proposición $A \wedge B$; el subconjunto $S_{A} \cup S_{B}$ representa la proposición $A \vee B$.

En el sistema de los dos interruptores podemos distinguir 16 proposiciones esencialmente diferentes, número que coincide con el número de 


\section{Introducción a la lógica cuántica}

subconjuntos $S$. Atendiendo al tipo de subconjunto que representa cada proposición, podemos clasificar las 16 proposiciones en 6 tipos:

1. La proposición representada por el espacio de fases completo es la proposición idénticamente verdadera, que representaremos por I. Siempre es verdadera. Hay muchas maneras de definirla (por ejemplo, $A \vee \neg A$, o $B \vee \neg B$ ), pero todas ellas son equivalentes (i.e., todas se representan por el mismo subconjunto, $S_{A} \cup \bar{S}_{A}=S_{B} \cup \bar{S}_{B}=S$ ).

2. Proposiciones representadas por tres cuadrantes de $S$. Son cuatro: $A \vee B$ (representada por $S_{A} \cup S_{B}$ ), $A \vee \neg B$ (representada por $S_{A} \cup \bar{S}_{B}$ ), $\neg A \vee B$ (representada por $\bar{S}_{A} \cup S_{B}$ ), y $\neg A \vee \neg B$ (representada por $\bar{S}_{A} \cup \bar{S}_{B}$ ).

3. Proposiciones representadas por dos cuadrantes opuestos de $S$. Son dos: $(A \vee B) \wedge(\neg A \vee \neg B)\left[\right.$ representada por $\left.\left(S_{A} \cup S_{B}\right) \cap\left(\bar{S}_{A} \cup \bar{S}_{B}\right)\right]$ y $(A \vee \neg B) \wedge(\neg A \vee B)$ [representada por $\left.\left(S_{A} \cup \bar{S}_{B}\right) \cap\left(\bar{S}_{A} \cup S_{B}\right)\right]$.

4. Proposiciones representadas por dos cuadrantes contiguos de $S$. Son proposiciones que especifican completamente el estado de uno de los dos interruptores y no dicen nada sobre el estado del otro. Son cuatro: $A$ (representada por $S_{A}$ ), $\neg A$ (representada por $\bar{S}_{A}$ ), $B$ (representada por $\left.S_{B}\right)$, y $\neg B$ (representada por $\bar{S}_{B}$ ).

5. Proposiciones representadas por un cuadrante de $S$. Son las proposiciones que especifican completamente el estado de ambos interruptores. Se llaman también proposiciones atómicas. Son cuatro: $A \wedge B$ (representada por $S_{A} \cap S_{B}$ ), $A \wedge \neg B$ (representada por $S_{A} \cap \bar{S}_{B}$ ), $\neg A \wedge B$ (representada por $\bar{S}_{A} \cap S_{B}$ ), y $\neg A \wedge \neg B$ (representada por $\bar{S}_{A} \cap \bar{S}_{B}$ ).

6. La proposición representada por el conjunto vacío, $\varnothing$, es la proposición idénticamente falsa. Es siempre falsa. Hay muchas maneras equivalentes de formularla; por ejemplo, $A \wedge \neg A$ (representada por $\left.S_{A} \cap \bar{S}_{A}=\varnothing\right)$ o $B \wedge \neg B$ (representada por $\left.S_{B} \cap \bar{S}_{B}=\varnothing\right)$.

Esta identificación entre proposiciones y subconjuntos se puede llevar aún más lejos. Entre los diferentes subconjuntos del espacio de fases se puede definir una relación de inclusión. Por ejemplo, $\left(S_{A} \cap S_{B}\right) \subset S_{A}$. Paralelamente, existe una relación de «inclusión" entre las propiedades del sistema: la propiedad «encendidos» de los dos interruptores es un caso particular de la propiedad «encendido» del primer interruptor. Por último, existe además una relación paralela de implicación lógica entre las correspondientes proposiciones: la proposición $A \wedge B$ («el primer interruptor está encendido y el segundo interruptor está encendido») «implica» la proposición $A$ («el primer interruptor está encendido»). Pongamos otro ejemplo. La relación entre conjuntos $S_{A} \subset\left(S_{A} \cup S_{B}\right)$, se traduce en la relación entre propiedades: la propiedad «encendido" del primer interruptor es un caso particular de la propiedad «en- 
cendido» de al menos uno de los interruptores; y en una relación entre proposiciones: $A$ («el primer interruptor está encendido) implica $A \vee B$ («el primer interruptor está encendido o el segundo interruptor está encendido»).

Diremos que una proposición $P$ implica otra $Q$, o abreviadamente $P \succeq Q$, cuando $Q$ es verdadera en todos los estados en los que $P$ es verdadera.

Con todo esto lo que queremos subrayar es que hay al menos tres descripciones diferentes de un sistema físico (espacio de fases, propiedades, proposiciones), que comparten una estructura común. Esa estructura «contiene»:

(i) Las relaciones de inclusión entre todos los subconjuntos del espacio de fases.

(ii) Las relaciones de «inclusión» entre todas las propiedades del sistema.

(iii) Las relaciones lógicas entre todas las proposiciones que se pueden formular sobre ese sistema.

En ese sentido, esa estructura común contiene «lo esencial» de ese sistema descrito por la física clásica. Esa estructura es una estructura algebraica. El objetivo de la lógica aplicada a una teoría física es encontrar las estructuras que "capturan» lo esencial de la teoría.

\section{B. Álgebras booleanas}

La estructura algebraica característica del conjunto de las proposiciones de cualquier sistema clásico es un álgebra booleana.

Diremos que $Z$ es un álgebra booleana si $Z=\{S, \vee, \wedge, \neg\}$, donde $S$ es un conjunto que contiene al menos dos elementos $(S$ y $\varnothing), \vee$ y $\wedge$ son operaciones entre dos elementos de $S$, y $\neg$ es una operación sobre un elemento de $S$, que satisfacen las siguientes identidades, $\forall A, B, C \in S$,

$$
\begin{aligned}
A \vee B & =B \vee A, \\
A \wedge B & =B \wedge A, \\
A \vee(B \vee C) & =(A \vee B) \vee C, \\
A \wedge(B \wedge C) & =(A \wedge B) \wedge C, \\
A \vee(A \wedge B) & =A, \\
A \wedge(A \vee B) & =A, \\
A \wedge(B \vee C) & =(A \wedge B) \vee(A \wedge C), \\
A \vee(B \wedge C) & =(A \vee B) \wedge(A \vee C), \\
A \vee(B \wedge \neg B) & =A, \\
A \wedge(B \vee \neg B) & =A .
\end{aligned}
$$




\section{Introducción a la lógica cuántica}

Esta definición de álgebra booleana, a costa de una cierta redundancia, muestra claramente la simetría entre las operaciones $\vee$ y $\wedge$. Las identidades (1a) y (1b) dicen que $\vee$ y $\wedge$, respectivamente, son conmutativas. (1c) y (1d) dicen que $\vee \mathrm{y} \wedge$, respectivamente, son asociativas. (1e) y (1f) se conocen como axiomas de absorción. (1g) indica que $\wedge$ es distributiva respecto $a \vee$; (1h) indica que $\vee$ es distributiva respecto a $\wedge$. (1i) y (1j) contienen las propiedades de la operación $\neg$.

En particular, el álgebra booleana característica del sistema formado por los dos interruptores se llama $\boldsymbol{Z}_{16}$, donde el subíndice señala el número de elementos (proposiciones esencialmente diferentes) que forman el conjunto.

Una propiedad de esta estructura, muy importante para la lógica, es la siguiente: Cualquier álgebra booleana $\mathcal{Z}=\{S, \vee, \wedge, \neg\}$ admite siempre una función $f: S \rightarrow\{0,1\}$ tal que $\forall A, B \in S$,

$$
\begin{aligned}
f(A \vee B) & =f(A) \vee f(B), \\
f(A \wedge B) & =f(A) \wedge f(B), \\
f(\neg A) & =\neg f(A) .
\end{aligned}
$$

Si interpretamos que 0 y 1 significan «falso" y «verdadero», entonces cada una de estas funciones $f$ proporciona una manera sistemática de asignar estos valores a todas las proposiciones del álgebra booleana. Por ejemplo, $\boldsymbol{Z}_{16}$ sólo admite cuatro funciones de ese tipo. Cada una de ellas corresponde a un estado posible del sistema.

\section{La lógica de la física cuántica}

\section{A. La lógica de Birkhoff y von Neumann}

En esta sección supondremos cierta familiaridad con el formalismo habitual de la MC. Los objetos básicos de la MC son el espacio de Hilbert $¥$ de los estados y sus operadores autoadjuntos asociados. El objeto básico del enfoque lógico-algebraico es la estructura algebraica del conjunto de todas las proposiciones del sistema. Esta estructura no es unica, pues depende de qué se elija como proposiciones y cómo se definan las operaciones entre ellas. La primera LC es obra de Birkhoff y von Neumann [4], y es en la que se han basado la mayor parte de los desarrollos posteriores.

Birkhoff y von Neumann consideran que las proposiciones cuánticas (también llamadas preguntas si-no, preguntas experimentales, o eventos cuánticos) son los elementos del conjunto formado por todos los subespa- 


\section{Adán Cabello}

cios cerrados del espacio de Hilbert, conjunto que denotaremos por $S(\not)$ ). Este conjunto es equivalentemente al de todos los proyectores sobre $\#$. Sobre este conjunto, Birkhoff y von Neumann proponen las siguientes definiciones, en analogía con las operaciones de la lógica clásica.

1. Una proposición $A$ implica otra $B, A \geq B$, si $\forall$ estado $\vec{\psi}$ tal que $\operatorname{Prob}_{\vec{\psi}}(A)=1$ ocurre que $\operatorname{Prob}_{\vec{\psi}}(B)=1$; i.e., $B$ es cierta en todos los estados en los que $A$ es cierta. Esto es equivalente a que $Z_{A}$ sea un subespacio cerrado de $\nVdash_{B}$. El análogo clásico es $S_{A} \in S_{B}$. Si $\hat{P}_{A}$ y $\hat{P}_{B}$ son los proyectores asociados, $\mathrm{A} \geq \mathrm{B}$ es equivalente a

$$
\hat{P}_{A} \hat{P}_{B}=\hat{P}_{B} \hat{P}_{A}=\hat{P}_{A},
$$

que se denota por $\hat{P}_{A} \succeq \hat{P}_{B} . A$ y $B$ son equivalentes si $\mathrm{A} \succeq B$ y $B \succeq A$.

2. La proposición idénticamente falsa corresponde con el operador nulo $\hat{0}$, que transforma cualquier vector de $\sharp$ en el vector nulo $\overrightarrow{0}$. La proposición idénticamente verdadera corresponde con el operador identidad $\hat{I}$, que deja invariante cualquier vector de $\mathcal{H}$. Cualquier proyector $\hat{P}_{A}$ satisface $\hat{0} \geq \hat{P}_{A} \geq \hat{I}$. El análogo clásico es $\varnothing \in S_{A} \in S$.

3. Si $A$ es una proposición, la proposición $\neg A$ (no $A$ ) se define exigiendo que $\forall$ estado $\vec{\psi}$, $\operatorname{Prob}_{\vec{\psi}}(\neg A)=1 \Leftrightarrow \operatorname{Prob}_{\vec{\psi}}(A)=0$. Esto es equivalente a $\sharp_{\neg A}=\left(\oiint_{A}\right)^{\perp}$, lo cual determina un único proyector $\hat{P}_{\neg A}$ que representa a $\neg A$ :

$$
\hat{P}_{\neg A}=\hat{I}-\hat{P}_{A},
$$

lo cual implica que $\forall \vec{\psi}$

$$
\operatorname{Prob}_{\vec{\psi}}(A)+\operatorname{Prob}_{\vec{\psi}}(\neg A)=1 .
$$

El análogo clásico es $S_{\neg A}=S-S_{A}$. Al contrario de lo que ocurre en el caso clásico, la definición cuántica tiene la peculiar propiedad de que $A$ puede ser falsa en un estado $\vec{\psi}$ sin que ello implique que $\neg A$ sea cierta. $A$ falsa quiere decir $\operatorname{Prob}_{\vec{\psi}}(A)<1 ; \neg A$ cierta quiere decir $\operatorname{Prob}_{\vec{\psi}}(A)=0$. Esta peculiaridad proviene de: (i) la existencia de superposiciones lineales de vectores propios de $\hat{P}_{A}$; y (ii) la insistencia en tener una lógica bivaluada (verdadero-falso), en lugar de una trivaluada (verdadero-falso-indeterminado).

4. La proposición $A \wedge B$ se define exigiendo que $\operatorname{Prob}_{\vec{\psi}}(A \wedge B)=1$ en un estado $\vec{\psi} \Leftrightarrow \operatorname{Prob}_{\vec{\psi}}(A)=1$ y $\operatorname{Prob}_{\vec{\psi}}(B)=1$. Esto quiere decir, 


$$
\mathbb{F}_{A \wedge B}=\mathbb{F}_{A} \cap \mathbb{F}_{B} \text {. }
$$

Esta definición resulta bastante natural expresada en términos de subespacios. Sin embargo, no hay una manera sencilla de trasladarla al lenguaje de los proyectores, ya que no existe una forma sencilla de escribir el proyector sobre el subespacio $\mathcal{Z}_{A \wedge B}$ en términos de los proyectores $\hat{P}_{A}$ y $\hat{P}_{B}$. En el caso particular de que $\hat{P}_{A}$ y $\hat{P}_{B}$ sean conmutativos, el proyector que representa la proposición $A \wedge B$ es

$$
\hat{P}_{A} \wedge \hat{P}_{B}=\hat{P}_{A} \hat{P}_{B}
$$

El análogo clásico es $S_{A \wedge B}=S_{A} \cap S_{B}$.

5. La proposición $A \vee B$ se define como el proyector sobre el menor subespacio lineal cerrado que contiene tanto a $\oiint_{A}$ como a $\oiint_{B}$,

$$
\oiint_{A \vee B}:=\overline{\oiint_{A} \oplus{\varkappa_{B}}_{B}}
$$

A primera vista, una definición más razonable de $A \vee B$ sería el proyector sobre el subespacio $\oiint_{A} \cup \mathcal{Z}_{B}$; sin embargo, éste no es un subespacio líneal de $Z$. Otra posible definición de $A \vee B$ podría haber sido el proyector sobre el subespacio $\mathcal{Z}_{A} \oplus \mathcal{Z}_{B}$; sin embargo, en el caso de $\mathbb{Z}^{\text {infinito, }}$ $\nVdash_{A} \oplus \nVdash_{B}$ puede no ser un subespacio cerrado. Como en el caso de $A \wedge B$, no hay una manera sencilla de escribir el proyector sobre $\nVdash_{A \vee B}$. En el caso particular de que $\hat{P}_{A}$ y $\hat{P}_{B}$ sean conmutativos, el proyector que representa la proposición $A \vee B$ es

$$
\hat{P}_{A} \vee \hat{P}_{B}=\hat{P}_{A}+\hat{P}_{B}-\hat{P}_{A} \hat{P}_{B}
$$

El análogo clásico es $S_{A \vee B}=S_{A} \cup S_{B}$.

El hecho de que dos observables cuánticos no sean siempre compatibles (i.e., que no se puedan medir simultáneamente sin perturbarse), que se traduce en el formalismo matemático en que los correspondientes operadores autoadjuntos no conmutan, se refleja en que la estructura algebraica del conjunto de las proposiciones asociada con las definiciones anteriores de $\wedge \mathrm{y} \vee$ no es distributiva. Esto se puede ver de una manera sencilla con ayuda de un ejemplo. Sean $\boldsymbol{H}_{\vec{\psi}_{1}}$ y $\boldsymbol{Z}_{\vec{\psi}_{2}}$ los subespacios unidimensionales de un espacio de Hilbert bidimensional $\mathbb{Z}$, subtendidos por (i.e., formados por todos los múltiplos complejos de) los vectores linealmente independientes $\vec{\psi}_{1}$ y $\vec{\psi}_{2}$, respectivamente, y $\vec{\phi}$ cualquier combinación lineal 
no trivial de $\vec{\psi}_{1}$ y $\vec{\psi}_{2}$. Entonces $\boldsymbol{Z}_{\vec{\phi}} \cap\left(\mathcal{H}_{\vec{\psi}_{1}} \oplus \mathcal{H}_{\vec{\psi}_{2}}\right.$ ) es simplemente $\boldsymbol{Z}_{\vec{\phi}}$ (porque $\left.\mathcal{H}_{\vec{\psi}_{1}} \oplus \mathcal{H}_{\vec{\psi}_{2}}=\mathcal{H}^{2}\right)$, mientras que $\left(\mathcal{H}_{\vec{\phi}} \cap \mathcal{H}_{\vec{\psi}_{1}}\right) \oplus\left(\mathcal{H}_{\vec{\phi}} \cap \mathcal{H}_{\vec{\psi}_{2}}\right)$ es el subespacio nulo (porque los subespacios a ambos lados del $\oplus$ son nulos).

El origen de muchas de las propiedades extrañas, no clásicas, de la MC puede rastrearse hasta esta propiedad no-distributiva de la estructura lógica de las proposiciones cuánticas.

\section{B. Estructuras algebraicas de la lógica cuántica}

Ya hemos visto qué la estructura algebraica de $S(\not{H})$ no es distributiva, pero todavía no sabemos cuál es su estructura característica. Para ello necesitaremos una serie de definiciones previas.

$A=\{S, \leq\}$ es un conjunto parcialmente ordenado (poset) si $S$ es un conjunto no vacío y $\leq$ es una relación reflexiva, transitiva y antisimétrica entre algunos elementos de $S$ (pero no necesariamente todos). Si tal relación existe entre cualquier pareja de elementos de $S$, se dice entonces que $A$ es un conjunto totalmente ordenado.

Si $A$ y $B$ son elementos de $S$, puede existir otro elemento $C$ tal que

$$
\begin{gathered}
A \leq C \text { y } B \leq C ; \\
\text { si } A \leq D \text { y } B \leq D \Rightarrow C \leq D .
\end{gathered}
$$

El elemento $C$ es el supremo de $\{A, B\}$, que equivale a $A \vee B$. De igual manera puede existir un elemento $E$ tal que

$$
\begin{gathered}
E \leq A \text { y } E \leq B ; \\
\text { si } F \leq A \text { y } F \leq B \Rightarrow F \leq E .
\end{gathered}
$$

El elemento $E$ es el ínfimo de $\{A, B\}$, que equivale a $A \wedge B$.

Un poset puede tener un elemento máximo, 1, o un elemento mínimo, 0 , o ambos, tales que $\forall A \in S$,

$$
\begin{aligned}
& 0 \leq A, \\
& A \leq 1
\end{aligned}
$$

Un poset se dice complementado si tiene un máximo, un mínimo, y $\forall A \in S \exists A^{\perp} \in S$ tal que

$$
\begin{aligned}
& A \vee A^{\perp}=1, \\
& A \wedge A^{\perp}=0 .
\end{aligned}
$$




\section{Introducción a la lógica cuántica}

Un poset se dice ortocomplementado si es complementado y $\forall A \in S$,

$$
\begin{gathered}
\left(A^{\perp}\right)^{\perp}=A, \\
A \leq B \Rightarrow B^{\perp} \leq A^{\perp} .
\end{gathered}
$$

Entre los elementos de un poset ortocomplementado se puede definir una relación de ortogonalidad mediante la siguiente condición:

$$
A \perp B \Leftrightarrow A \leq B^{\perp} \text {. }
$$

Llamaremos identidad ortomodular a

$$
A \leq B \Rightarrow B=A \vee\left(B \wedge A^{\perp}\right) .
$$

Un poset $\mathcal{A}$ es ortocompleto si es ortocomplementado y cada pareja de subconjuntos de $S$ mutuamente ortogonales tiene un supremo.

Un poset $\mathscr{A}$ es ortomodular si es ortocompleto y cumple la identidad ortomodular.

Un poset $\mathscr{A}$ es un retículo si cada pareja de elementos de $S$ tiene un supremo y un ínfimo. Cualquier retículo satisface las propiedades conmutativas, asociativas y de absorción de un álgebra booleana (pero no necesariamente las propiedades distributivas). Si además satisface las propiedades distributivas diremos que se trata de un retículo distributivo. Un retículo distributivo ortocomplementado es un retículo booleano o álgebra booleana.

Con este vocabulario ya podemos decir cuál es la estructura del conjunto de las proposiciones cuánticas con las definiciones propuestas por Birkhoff y von Neumann. $S(\mathcal{H})$ es un retículo, parcialmente ordenado por inclusión, y tal que para cualquier pareja de subespacios existe un subespacio mayor que es común a ambos, y un subespacio menor que los contiene a ambos. \# es el máximo y el subespacio nulo es el mínimo. El cierre del conjunto de vectores ortogonal a un cierto subespacio forma el correspondiente subespacio ortogonal, que es el ortocomplemento del subespacio original. Por tanto, $S(\mathcal{H})$ forma un retículo ortocomplementado pero no distributivo.

Otra caracterización alternativa de la estructura algebraica de $S\left(\Re^{\prime}\right)$ se obtiene tras las siguientes definiciones.

Sea $\mathbb{M}=\left\{\mathcal{B}_{i}: i \in N\right\}$ una familia numerada (y puede que infinita) de álgebras booleanas: $\mathcal{B}_{i}=\{S, \vee, \wedge, \neg\}$. $\mathcal{M}$ es una variedad booleana

$$
\text { si } i, j \in N \Rightarrow \exists k \in N \text { tal que } S_{i} \cap S_{j}=S_{k} \text {; }
$$




\section{Adán Cabello}

$$
\begin{aligned}
\forall i, j & \in N, \\
0_{i} & =0_{j} \mathrm{y} \\
1_{i} & =1_{j} ;
\end{aligned}
$$

si $A, B \in S_{i} \cap S_{j} \Rightarrow$

$$
\begin{aligned}
A \vee_{i} B & =A \vee_{j} B, \\
A \wedge_{i} B & =A \wedge_{j} B, \\
\neg_{i} A & =\neg_{j} B .
\end{aligned}
$$

Mes un álgebra parcialmente booleana si es una variedad booleana y $\forall A$, $B, C \in \cup\left\{S_{i}\right\}$, si $\exists i, j, k \in N$ tal que $A, B \in S_{i} ; B, C \in S_{j} ; C, A \in S_{k} \Rightarrow \exists m \in N$ tal que $A, B, C \in S_{m}$.

En resumen, un álgebra parcialmente booleana es un conjunto de álgebras booleanas pegadas juntas de una forma consistente, de manera que cuando se superponen dos o más de ellas, sus operaciones están en concordancia entre sí. $S(\mathbb{Z})$ también es un álgebra parcialmente booleana.

\section{4. Éxitos y fracasos de la lógica cuántica}

\section{A. El teorema de Kochen-Specker}

En 1960, en un breve artículo titulado «La lógica de las proposiciones que no son decidibles simultáneamente" [36], Specker se pregunta: «¿puede ampliarse la descripción de un sistema mecanocuántico (...) de manera que la lógica clásica de las proposiciones sea válida en el dominio ampliado?». Pregunta que, en el lenguaje introducido en la sección anterior, es equivalente a "ipuede ampliarse un álgebra parcialmente booleana de manera que sea inmersible en un álgebra booleana?». Specker contesta: «La respuesta a esta pregunta es negativa excepto en el caso de espacios de Hilbert (...) de dimensiones 1 y $2 »$. La imposibilidad de tal inmersión para espacios de más de dos dimensiones es consecuencia del hecho de que no existe tal inmersión en el caso tridimensional. Tal inmersión requeriría que fuese posible establecer una correspondencia $f$ entre el conjunto de todos los subespacios de un espacio vectorial tridimensional y un álgebra booleana, de manera que para cada pareja $A, B$ de subespacios: 


$$
\begin{aligned}
f(A \wedge B) & =f(A) \wedge f(B), \\
f(A \vee B) & =f(A) \vee f(B), \\
f(\varnothing) & =0, \\
f(\not) & =1 .
\end{aligned}
$$

El álgebra booleana de dos elementos (aquélla en la que $S=\{\varnothing, S\}$ ) es la imagen homeomórfica de cualquier álgebra booleana. Eso hace que sólo exista esa inmersión si es posible asignar uno de los valores 0 («falso») y 1 «verdadero») a todos los subespacios lineales de un espacio vectorial tridimensional, de manera que: (i) al espacio completo se le asigne 1 , y al espacio nulo 0; (ii) si $A$ y $B$ son subespacios ortogonales, entonces a su intersección $A \wedge B$ se le asigna el valor 1 sólo si $A$ y $B$ valen 1 ; y (iii) a su combinación lineal, $A \vee B$, se le asigna el valor 1 , si el valor 1 se le asigna al menos a uno de sus subespacios $A, B$. Specker concluye: «Un argumento geométrico elemental muestra que tal asignación es imposible». Siete años más tarde, en colaboración con Kochen, publicaron el primer ejemplo explícito de un conjunto de ( 1117 !) subespacios unidimensionales de un espacio tridimensional sobre el que es imposible completar tal asignación [22].

Esta imposibilidad es lo que se conoce como el teorema de KochenSpecker, que se puede formular como sigue: $\mathrm{Si}\left\{A_{i}\right\}$ es un conjunto de $d$ subespacios lineales unidimensionales mutuamente ortogonales de un espacio de Hilbert $\mathbb{Z}$ de dimensión $d \geq 3$, no existe una función $f$ entre $S(\mathcal{H})$ y el álgebra booleana $Z_{2}$ (la formada sólo por los elementos 0 y 1 ) tal que:

$$
\begin{gathered}
\bigcup_{i=1}^{d} f\left(A_{i}\right)=f\left(\bigcup_{i=1}^{d} A_{i}\right)=f(\mathcal{Z})=1 \mathrm{y} \\
f\left(A_{i}\right) \cap f\left(A_{j}\right)=f\left(A_{i} \cap A_{j}\right)=f(\varnothing)=0, \forall i \neq j .
\end{gathered}
$$

Lo que, en términos de proyectores equivale a decir que dado un conjunto de proyectores unidimensionales mutuamente ortogonales, la función $f$ debe asignar el valor 1 (»verdadero») a exactamente uno de esos proyectores, y el valor cero («falso») a los otros $d-1$.

La demostración más sencilla de que tal asignación es imposible requiere sólo 18 subespacios unidimensionales de un espacio tetradimensional [5-7]. Está contenida en la siguiente tabla:

$$
\begin{array}{lllllllllll}
1000 & 1111 & 1111 & 1000 & 1001 & 1001 & 111 \overline{1} & 111 \overline{1} & 100 \overline{1} \\
0100 & 1 \overline{1} \overline{1} & \overline{1} \overline{1} 1 \overline{1} & 0010 & 0100 & \overline{1} \overline{1} \overline{1} & \overline{1} \overline{1} 00 & 0101 & 0110 \\
0011 & 1 \overline{1} 00 & 10 \overline{1} 0 & 0101 & 0010 & 11 \overline{1} \overline{1} & 0011 & 10 \overline{1} 0 & 11 \overline{1} 1 \\
001 \overline{1} & 001 \overline{1} & 010 \overline{1} & 010 \overline{1} & 100 \overline{1} & 0110 & 11 \overline{1} 1 & \overline{1} \overline{1} 11 & 1 \overline{1} 11
\end{array}
$$


La tabla consta de 9 columnas, en cada columna hay 4 vectores mutuamente ortogonales. En total hay 36 vectores pero sólo 18 distintos. La notación es la siguiente: $001 \overline{1}$ representa el vector (normalizado) $\frac{1}{\sqrt{2}}(0,0,1,-1)$. Cada vector representa el proyector sobre el subespacio engendrado por ese vector. Es imposible asignar los valores uno y cero siguiendo las reglas mencionadas antes. Para verlo, sólo hay que fijarse en que cada vector aparece exactamente en dos columnas, de manera que el número total de unos debería ser par, mientras que el número de columnas (y por tanto, de unos) es nueve.

Este resultado se interpreta habitualmente diciendo que los experimentos sobre un sistema cuántico no tienen resultados predefinidos. $\mathrm{O}$ más exactamente, que tales resultados, si estuviesen predefinidos serían contextuales; es decir, que el resultado de medir el proyector $A_{i}$ dependería de si se mide conjuntamente con unos u otros proyectores.

Recientemente se ha cuestionado el teorema de Kochen-Specker en base al siguiente resultado: si bien la asignación es imposible para el conjunto de todos los subespacios unidimensionales, sí es posible para ciertos subconjuntos densos de subespacios [12]. Esto se interpreta de la siguiente manera: dado que la precisión de cualquier medición es necesariamente finita, ningún experimento permitiría distinguir entre el conjunto total y esos subconjuntos densos; por tanto, es posible que la naturaleza "conspire» de manera que cuando se le pregunta por alguna de las proposiciones no incluidas en el conjunto denso, ella da la respuesta predefinida a una pregunta distinta (pero infinitamente próxima) [25, 21]. De hecho, ya se han construido modelos explícitos en los que casi todas las proposiciones tienen resultados predefinidos y cuyas predicciones son indistinguibles de las de la MC [8].

\section{B. El programa de las escuelas de Harvard y Ginebra}

Bajo el nombre de enfoque axiomático de la LC se reúnen toda una serie de trabajos de Mackey [23], Maczyński [24], Finkelstein [9], Jauch [20], y Piron [26,27] que esencialmente son una elaboración de las ideas de Birkhoff y von Neumann. La pregunta inicial que se hacen estos autores es la siguiente: En el supuesto de que todas las características del espacio de Hilbert (que lo son también de la $\mathrm{MC}$ ) puedan quedar reflejadas en una estructura algebraica; esto es, que fuese posible una reconstrucción algebraica completa de la MC. ¿Qué se ganaría con esa reformulación? Una posibilidad atractiva consistiría en que se obtuviese una mejor perspectiva de cuál es la relación de la MC tanto con la mecánica 


\section{Introducción a la lógica cuántica}

clásica como con aquellas hipotéticas teorías que fuesen a suceder a la MC. ¿Pero cómo se puede decir algo útil sobre una teoría que todavía no se ha formulado? Una forma de aproximarse a este proyecto es considerar la siguiente pareja de problemas relacionados: (I) ¿Existen restricciones algebraicas sobre el conjunto de los sucesos experimentales que deban ser satisfechas a priori por cualquier teoría física? (II) ¿Qué restricciones adicionales, peculiares de cada teoría, dan lugar a que la estructura del conjunto de los sucesos experimentales de la mecánica clásica sea un álgebra booleana, y la de la MC sea una estructura no-booleana?

Para responder estas preguntas consideremos el conjunto de todos los "eventos experimentales" $\varepsilon$ de una teoría física, y llamemos $\varepsilon_{A}$ al conjunto de los eventos asociados a un experimento específico $A$. Es fácil ver que la estructura de $\varepsilon_{A}$ es un álgebra booleana $\mathcal{B}_{A}$, cuyas operaciones son "unión», «intersección» y "complementación», y cuyos elementos mínimo y máximo son, respectivamente, el «evento nulo" (el conjunto vacío) y el «evento seguro» (el conjunto de todos los posibles resultados de $A$ ). A cada elemento de $\varepsilon_{A}$ será posible asignarle una respuesta "sí" (el equivalente a "verdadero») o «no» («falso») cuando se haga el experimento $A$. Por tanto, $\varepsilon$ es una familia de álgebras booleanas que están en correspondencia uno-a-uno con el conjunto formado por los posibles experimentos. Estos autores concluyen que la respuesta a (I) es que la estructura del conjunto de los eventos de cualquier teoría física es una ortoálgebra (cuya definición omitiremos). Algunos de ellos llegan más lejos y afirman que además es un retículo ortocomplementado [20,9,26], pero esto ofrece ciertas dificultades ([17], p. 199).

Asimismo, la respuesta de estos autores a (II) es que, haciendo ciertas hipótesis de carácter general, la estructura del conjunto de los eventos experimentales de cualquier teoría que haga referencia a los valores de observables físicos es un poset ortomodular. Para más detalles consúltense las referencias mencionadas.

Si bien una reformulación de la $\mathrm{MC}$ en estos términos añade algo a nuestro conocimiento de la teoría, no parece aportar nada al problema de la interpretación. Dicho de otra manera, los elementos del álgebra booleana se pueden interpretar de una manera diferente según la interpretación de la MC que uno adopte. Véase, por ejemplo, [18], Sec. 9.2.1. En el mejor de los casos, suponiendo que tal reformulación fuese completa, no está claro porqué ésta debería enseñarnos algo nuevo. Además tal reformulación por ahora es sólo parcial, por dos motivos: (a) No se ha encontrado una forma puramente algebraica de especificar los retículos ortomodulares (o álgebras booleanas parciales) que son isomorfas a $S(\mathcal{H})$. (b) 


\section{Adán Cabello}

No se ha encontrado nada en esta reformulación que juegue el papel de principio dinámico; la LC todavía no tiene el equivalente de la ecuación de Schrödinger.

\section{Las expectativas de Putnam}

La LC suscitó toda una serie de expectativas sobre futuros desarrollos basados en ella. Probablemente las más ambiciosas son las sugeridas por Putnam en [29]:

(1) La lógica es una ciencia empírica; algunas de las «verdades necesarias» de la lógica clásica podrían volverse falsas por razones empíricas ([29], pp. 216, 226).

(2) De la misma manera que la teoría general de la relatividad nos lleva a adoptar una geometría no-euclidiana, nuestra mejor interpretación de la MC nos debe llevar a adoptar una lógica no-clásica (p. 234).

(3) Adoptando una lógica no-clásica podemos retener buena parte de las propiedades de un sistema (p. 229).

A día de hoy la sugerencia (1) «se ha convertido en una tesis mucho menos revolucionaria de lo que uno podría pensar» ( [17], p. 209, y Sec. 7.9). La analogía que se propone en (2) sigue siendo sugestiva, pero depende de qué se entienda por una «lógica no-clásica». La tesis (3), aún asumiendo el término «lógica no-clásica» en un sentido amplio, ha resultado ser falsa ([38], p. 588; [17], Secs. 7.8 y 7.9; [32], Sec. 7.5), esencialmente por culpa del teorema de Kochen-Specker. De hecho, la postura de Putnam ha cambiado desde entonces [11, 31].

\section{Pequeña guía bibliográfica del laberinto de la lógica cuántica}

En este trabajo nos hemos centrado en un tipo particular de LC y no distributiva, cuyo origen es el artículo de Birkhoff y von Neumann [4] (ver también los trabajos de Putnam [29,30]), que es la base de la mayoría de los desarrollos posteriores. Para terminar, nos gustaría añadir algunas referencias sin las cuales es imposible obtener una idea clara de la amplitud y variedad de la LC.

Los libros de Hooker $[14,15]$ recogen buena parte de los artículos originales sobre LC. El desarrollo histórico (hasta 1974) de las diversas variedades de la LC se trata en el capítulo 8 del libro de Jammer [19]. En- 


\section{Introducción a la lógica cuántica}

tre las otras variedades de la LC, que gozan de menos predicamento, cabe destacar la lógica trivaluada, en la que cada proposición puede ser "verdadera", "falsa», o «indeterminada». Esta idea (que no está exenta de dificultades) fue propuesta por Reichenbach [33] y desarrollada también por Putnam [28].

La literatura sobre los intentos basados en la LC para resolver el problema de la interpretación de la MC es muy extensa. Como introducción consúltese [32], Cap. 7; y [17], Cap. 7.

Otras referencias introductorias a la LC son [35,40,41,2,13,34]. La más asequible, y la única con versión en español, es [16]. La más reciente es [39].

\section{Referencias}

1 J. S. BELL, "On the impossible pilot wave", Found. Phys. 12, 989-999 (1982). Reimpreso en Speakable and unspeakable in quantum mechanics, Cambridge University Press, Cambridge, 1987, pp. 159-168; y en M. Bell, K. GotTFried y M. Veltman (eds.), Quantum mechanics, high energy physics and accelerators: Selected papers of John Bell, World Scientific, River Edge, New Jersey, 1995. Versión en español: “Sobre la imposible onda piloto", en Lo decible y lo indecible en mecánica cuántica, Alianza, Madrid, 1990, pp. 221-233.

2 E. G. Beltrametti y B. van FraAssen (eds.), Current issues in quantum logic, Plenum, Press New York, 1981.

3 E. G. Beltrametti y G. Cassinelli, The logic of quantum mechanics, Addison-Wesley, Reading, Massachusetts, 1981.

4 G. Birkhoff y J. von NeumanN, "The logic of quantum mechanics", Ann. Math. 37, 823-843 (1936). Reimpreso en A. H. Taub (ed.), [J. von Neumann's] Collected works, Pergamon Press, New York, 1961, vol. 4, pp. 105-125; y en [14], pp. 1-26.

5 A. Cabello, J. M. Estebaranz y G. García AlCaine, "Bell-Kochen-Specker theorem: A proof with 18 vectors", Phys. Lett. A 212, 183-187 (1996).

6 A. CABello, "A proof with 18 vectors of the Bell-Kochen-Specker theorem", en M. Ferrero y A. van der Merwe (eds.), New developments on fundamental problems in quantum physics, Kluwer Academic, Dordrecht, 1997, pp. 59-62.

7 A. CABELlo, "Kochen-Specker theorem and experimental test on hidden variables", Int. J. Mod. Phys. A 15, 2813-2820 (2000).

8 R. K. Clifton y A. Kent, "Simulating quantum mechanics by non-contextual hidden variables", Proc. R. Soc. Lond. A 456, 2101-2114 (2000).

9 D. Finkelstein, "Matter, space and logic", en R. S. Cohen y M. W. Wartofsky (eds.). Boston studies in the philosophy of science. Vol. 5, Reidel, Dordrecht, 1969, pp. 199-215.

${ }^{10}$ D. Finkelstein, "The physics of logic", en R. G. Colodny (ed.), Paradigms and paradoxes. The philosophical challenge of the quantum domain, University of Pittsburgh Press, Pittsburgh, 1972, pp. 47-66. Reimpreso en |15|, pp. 141-160.

${ }^{11}$ M. Friedman y H. Putnam, "Quantum logic, conditional probability and inference", Dialectica 32, 305-315 (1978).

12 C. D. GoDSIL y J. ZAKS, "Colouring the sphere», University of Waterloo research report CORR 88-12 (1988). 


\section{Adán Cabello}

${ }^{13}$ D. G. Holdsworth y C. A. Hooke, "A critical survey of quantum logic", en Logic in the 20th Century. Scientia special issue, 127-246 (1983).

${ }^{14}$ C. A. Hooker (ed.), The logico-algebraic approach to quantum mechanics. Volume I. Historical evolution, Reidel, Dordrecht, 1975.

15 C. A. Hooker (ed.), The logico-algebraic approach to quantum mechanics. Volume II. Contemporary consolidation, Reidel, Dordrecht, 1979.

16 R. I. G. Hughes, «Quantum logic», Scientific American 245, 146-157 (1981). Versión en español: «Lógica cuántica», Investigación y Ciencia, Diciembre 1981, 80-94.

17 R. I. G. HugHES, The structure and interpretation of quantum mechanics, Harvard University Press, Cambridge, Massachusetts, 1989.

18 C. J. IsHaM, Lectures on quantum theory. Mathematical and structural foundations, Imperial College Press, World Scientific, Singapore, 1995.

19 M. JAMMER, The philosophy of quantum mechanics: The interpretations of quantum mechanics in historical perspective, Wiley, New York, 1974.

20 J. M. JAUCH, Foundations of quantum mechanics, Addison-Wesley, Reading, Massachusetts, 1968.

21 A. KENT, "Noncontextual hidden variables and physical measurements", Phys. Rev. Lett. 83, 3755-3757 (1999).

$22 \mathrm{~S}$. Kochen y E. P. SPECKER, "The problem of hidden variables in quantum mechanic", J. Math. Mech. 17, 59-87 (1967). Reimpreso en [14], pp. 293-328; y en [37], pp. 235-263.

${ }^{23}$ G. W. MaCKEY, Mathematical foundations of quantum mechanics, Benjamin, New York, 1963.

24 M. J. MACZYNSKI, "A remark on Mackey's axiom system for quantum mechanics", Bulletin de L'Académie Polonaise des Sciences, Serie des Sciences Mathematiques, Astronomiques et Physiques 15, 583-587 (1967).

${ }^{25}$ D. A. MEYER, "Finite precision measurement nullifies the Kochen-Specker theorem", Phys. Rev. Lett. 83, 3751-3754 (1999).

${ }^{26}$ C. Piron, "Survey of general quantum physics", Found. Phys. 2, 287-314 (1972).

${ }^{27}$ C. PIRON, Foundations of quantum physics, Benjamin, Reading, Massachusetts, 1976.

${ }^{28}$ H. Putnam, “Three-valued logic", Phil. Studies 8, 73-80 (1957).

${ }^{29}$ H. Putnam, "Is logic empirical?", en R. S. Cohen y M. W. Wartofsky (eds.), Boston studies in the philosophy of science. Vol. 5, Reidel, Dordrecht, 1969, pp. 216-241.

${ }^{30}$ H. PUTNAM, "How to think quanturn-logically", Synthese 29,55-61 (1974). Reimpreso en P. SupPes (ed.), Logic and probability in quantum mechanics, Reidel, Dordrecht, 1976.

${ }^{31}$ H. PutnaM, "Quantum mechanics and the observer", Erkenntnis 16, 193-219 (1981).

32 M. L. G. REDHEAD, Incompleteness, nonlocality, and realism, Oxford University Press, New York, 1987.

${ }^{33} \mathrm{H}$. REICHENBACH, Philosophical foundations of quantum mechanics, University of California Press, Berkeley, California, 1944.

34 I. PItowsky, Quantum probability-quantum logic, Springer-Verlag, Berlin, 1989. 1973.

$35 \mathrm{E}$. Scheibe, The logical analysis of quantum mechanics, Pergamon Press, Oxford,

36 E. P. SPECKER, “Die Logik nicht gleichzeitig entscheidbarer Aussagen", Dialectica 14, 239-246 (1960). Reimpreso en [37], pp. 175-182. Versión en inglés: “The logic of propositions which are not simultaneously decidable", en [14], pp. 135-140. 


\section{Introducción a la lógica cuántica}

37 E. P. SPECKer, Selecta, Birkhäuser Verlag, Basel, 1990.

38 A. STAIRS, “Quantum logic, realism and value definiteness", Philos. Sci. 50, 578-60 (1983).

${ }^{39}$ K. Svozil, Quantum logic, Springer-Verlag, Berlin, 1998.

40 B. C. van FraAsSEN, "Semantie analysis of quantum logic", in C. A. Hooker (ed.), Contemporary research in the foundations and philosophy of quantum theory, Reidel, Dordrecht, 1973, pp. 80-113.

41 B. C. VAN FraAsSen, "The labyrinth of quantum logic", en R. S. Cohen y M. W. Wartofsky (eds.), Logical and epistemological studies in contemporary physics. Boston studies in the philosophy of sciences. Vol. 13, Reidel, Dordrecht, 1974, pp. 72-102. Reimpreso en [14], pp. 577-607. 